\title{
REPRESENTATIONS OF NORMED ALGEBRAS WITH MINIMAL LEFT IDEALS
}

\author{
by BRUCE A. BARNES $\dagger$
}

(Received 20th August 1973)

\section{Introduction}

The theory of *-representations of Banach *-algebras on Hilbert space is one of the most useful and most successful parts of the theory of Banach algebras. However, there are only scattered results concerning the representations of general Banach algebras on Banach spaces. It may be that a comprehensive representation theory is impossible. Nevertheless, for some special algebras interesting and worthwhile results can be proved. This is true for $\mathscr{B}(Y)$, the algebra of all bounded operators on a Banach space $Y$, and for $\mathscr{F}(Y)$, the subalgebra of $\mathscr{B}(Y)$ consisting of operators with finite dimensional range. The representations of $\mathscr{B}(Y)$ are studied in a recent paper by $H$. Porta and $E$. Berkson (6), and in another recent paper (8), P. Chernoff determines the structure of the representations of $\mathscr{F}(Y)$ (and also of some more general algebras of operators). In both these papers, $\mathscr{F}(Y)$, which is the socle of the algebras under consideration, plays an important role in the theory. This suggests the possibility that a more general representation theory can be formulated in the case of a normed algebra with a nontrivial socle. This we attempt to do in this paper.

Let $\pi$ be a continuous representation of a normed algebra $A$ into $\mathscr{B}(X)$, $X$ a Banach space. We have several main concerns in this paper. First, when does there exist minimal closed $\pi$-invariant subspaces of $X$, and when they do exist, does $\pi$ restricted to such a subspace have some reasonable characterisation? Second, under what conditions does a minimal closed $\pi$-invariant subspace of $X$ have a closed $\pi$-invariant complement in $X$ ? Third, is it possible to determine the structure of $\pi$ in some reasonable way?

The first two of these questions are treated in Section 3. The answers to both questions involve a special property of the minimal idempotents of the algebra $A$. This property is the topic of Section 2. The third question is considered in Section 4. In that section, following a pattern established by P. Chernoff in (8), we determine the structure of $\pi$ restricted to certain closed $\pi$-invariant subspaces of $X$. The subrepresentations of $\pi$ of this kind are then summed to $\pi$ in some special cases. There is a final very brief section concerning applications.

At this point we introduce our notation and conventions. All vector spaces

$\dagger$ This research was supported in part by NSF Grant GP-28250 A\#2 
in this paper are complex. Throughout this paper $A$ is a semisimple normed algebra and $X$ is a Banach space. We denote the norm on both spaces (and also some others) by $\|\cdot\|$. We assume throughout that $\pi$ is a continuous representation of $A$ into $\mathscr{B}(X)$. Minimal idempotents of $A$ are important in our considerations. Information concerning minimal idempotents, minimal left or right ideals, and the socle can be found in (18). If $e$ is a minimal idempotent (abbreviated m.i.) of $A$, then in our case, $e A e=\{\lambda e: \lambda$ complex $\}$. We denote the socle of an algebra $B$ by $S_{B}$.

We have already defined $\mathscr{B}(X)$ and $\mathscr{F}(X)$. We let $\mathscr{K}(X)$ denote the algebra of compact operators on $X$, and let $\mathscr{D}(X)$ denote the set of all operators in $\mathscr{B}(X)$ with range of dimension less than or equal to one. The dual space of $X$ is $X^{*}$ and the adjoint of an operator $T \in \mathscr{B}(X)$ is $T^{*}$. We denote the range of an operator $T$ by $\mathscr{R}(T)$. If $x \in X$ and $f \in X^{*}$, let $(f \mid x)$ be the operator defined on $X$ by $(f \mid x)(y)=f(y) x, y \in X$. If $x \in X$ and $f \in X^{*}$, we often use the notation $\langle x, f\rangle$ for $f(x)$.

A nonzero algebra of operators $B$ is irreducible on $X$ if there are no nontrivial closed $B$-invariant subspaces of $X . B$ is strictly irreducible if there are no non-trivial $B$-invariant subspaces of $X$. A representation $\pi: A \rightarrow \mathscr{B}(X)$ is irreducible (strictly irreducible) if $\pi(A)$ is irreducible (strictly irreducible) on $X$.

If $B$ is a collection of operators on $X$ and $Y$ is a subset of $X$, then

$$
B Y=\operatorname{span}\{T y: T \in B, y \in Y\} .
$$

Similarly, when $B$ and $C$ are subsets of $A$, then

$$
B C=\operatorname{span}\{b c: b \in B, c \in C\} .
$$

If $Y$ is a subset of a topological space $W$, then $\operatorname{cl}(Y)$ denotes the closure of $Y$ in $W$.

\section{Minimal idempotents that are full in an algebra}

Let $e$ be a m.i. of $A$. Then

$$
e A e=\{\lambda e: \lambda \text { complex }\} \text {. }
$$

We define a complex valued bilinear form $(.,$.$) on A e \times e A$ by $(a e, e b)=\lambda$, where $e b a e=\lambda e$. With respect to this bilinear form the two normed spaces $A e$ and $e A$ are in normed duality (see (18, Definition (2.4.8))).

Now we introduce a property that plays a central role in the study of certain representations of $A$. If $e$ is a m.i. of $A$, this property is that $e A$ determines the norm on $A e$ via the bilinear form $(.,$.$) . In order to formulate this property$ precisely, let

$$
\|a e\|_{1}=\sup \{|(a e, e b)|:\|e b\| \leqq 1\} .
$$

Definition 2.1. The m.i. $e$ is full in $A$ if there exists $K>0$ such that

$$
K\left\|_{i}^{*} a e\right\|_{1} \geqq\|a e\| \text { for all } a_{-}^{-} \in A \text {. }
$$

Note that for any m.i. $e$ of $A$,

$$
|(a e, e b)|\|e\|=\|e b a e\| \leqq\|e b\|\|a e\| .
$$


Therefore $e$ is full in $A$ if and only if the two norms $\|\cdot\|_{1}$ and $\|\cdot\|$ are equivalent on $A e$.

It is not difficult to find examples of algebras that contain m.i.'s that are not full in the algebra; see Example 2.9. However, there are many interesting examples of algebras in which every m.i. of the algebra is full. We give a number of examples in this section. Before providing specific examples, we prove a basic proposition.

Proposition 2.2. Let $A$ be a subalgebra of $\mathscr{B}(X)$ with the properties

(i) $A$ is irreducible on $X$,

(ii) $\mathscr{D}(X) \cap A$ is closed in $\mathscr{B}(X)$, and

(iii) $\mathscr{F}(X) \cap A \neq\{0\}$.

Then there exists a minimal algebra norm $\|.\|^{\prime}$ on $A$ (i.e., if $|$.$| is any algebra$ norm on $A$ there exists $J>0$ such that $J|a| \geqq\|a\|^{\prime}$ for all $a \in A$ ). Furthermore, every m.i. of $A$ is full in $\left(A,\|.\|^{\prime}\right)$.

Proof. By (5, Theorem 2), there is a closed total subspace $Y$ of $X^{*}$ such that

$$
\mathscr{D}(X) \cap A=\{(f \mid x): x \in X, f \in Y\} .
$$

Also, for all $T \in A, T^{*}(Y) \subset Y$, and $\left\{T^{*}: T \in A\right\}$ acts strictly irreducibly on $Y$. If $T \in A$, let

$$
\|T\|^{\prime}=\sup \left\{\left\|T^{*} f\right\|: f \in Y,\|f\| \leqq 1\right\} .
$$

It is easily verified that $\|\cdot\|^{\prime}$ is an algebra norm on $A$, and a direct application of (18, Theorem (2.4.17)) proves that $\|.\|^{\prime}$ is a minimal norm on $A$.

Now let $E$ be a m.i. of $A$. By (4, Lemma 1), $E=(f \mid x)$ for some $x \in X$ and $f \in Y$ such that $f(x)=1$. If $T, S \in A$, then

$$
\begin{gathered}
(T E, E S) E=E S T E=\left\langle T x, S^{*} f\right\rangle E, \\
E S=\left(S^{*} f \mid x\right), \text { and } T E=(f \mid T x) .
\end{gathered}
$$

Let $J=\sup \{|g(x)|: g \in Y,\|g\| \leqq 1$.$\} Then$

Also,

$$
\begin{aligned}
\|E S\|^{\prime} & =\sup \left\{\left\|(E S)^{*} g\right\|: g \in Y,\|g\| \leqq 1\right\} \\
& =\sup \left\{|g(x)|\left\|S^{*} f\right\|: g \in Y,\|g\| \leqq 1\right\} \\
& =J\left\|S^{*} f\right\| .
\end{aligned}
$$

Finally,

$$
\begin{aligned}
\|T E\|^{\prime} & =\sup \left\{\left\|(T E)^{*} g\right\|: g \in Y,\|g\| \leqq 1\right\} \\
& =\sup \{|g(T x)|\|f\|: g \in Y,\|g\| \leqq 1\} .
\end{aligned}
$$

$$
\begin{aligned}
\sup & \left\{|(T E, E S)|:\|E S\|^{\prime} \leqq 1\right\} \\
& =\sup \left\{\left|\left\langle T x, S^{*} f\right\rangle\right|: J\left\|S^{*} f\right\| \leqq 1\right\} \\
& =J^{-1} \sup \{|\langle T x, g\rangle|: g \in Y,\|g\| \leqq 1\} \\
& (J\|f\|)^{-1}\|T E\| !
\end{aligned}
$$


Corollary 2.3. Assume that $A$ is a subalgebra of $\mathscr{B}(X)$ and that the minimal left and right ideals of $A$ are complete subspaces of $A$. Assume further

$$
\mathscr{D}(X) \cap A=\{(f \mid x): x \in X, f \in Y\}
$$

where $Y$ is a closed norm determining subspace of $X^{*}$. Then every m.i. of $A$ is full in $A$.

Proof. Let $|$.$| denote the operator norm on \mathscr{B}(X)$, and let $\|\cdot\|^{\prime}$ be the minimal algebra norm on $A$ defined in the proposition. Since $\|\cdot\|^{\prime}$ is minimal on $A$, there exists $K>0$ such that $K\|T\| \geqq\|T\|^{\prime}$ for all $T \in A$. By hypothesis $Y$ is norm determining for $X$, so there exists $J>0$ such that

$$
\sup \{|f(x)|: f \in Y,\|f\| \leqq 1\} \geqq J\|x\|
$$

for all $x \in X$. Recalling the definition of $\|\cdot\|^{\prime}$, we have

$$
\begin{aligned}
\|T\|^{\prime} & =\sup \left\{\left\|T^{*} f\right\|: f \in Y,\|f\| \leqq 1\right\} \\
& =\sup \{|\langle T x, f\rangle|: x \in X, f \in Y,\|x\| \leqq 1,\|f\| \leqq 1\} \\
& \geqq J|T| .
\end{aligned}
$$

Thus $K\|T\| \geqq\|T\|^{\prime} \geqq J|T|$ for all $T \in A$, and $|$.$| and \|\cdot\|^{\prime}$ are equivalent norms on $A$.

Now let $E=(f \mid x)$ be a m.i. of $A$. Then $A E$ and $E A$ are complete subspaces of $(A,||$.$) and of (A,\|\cdot\|)$. Since the spaces $(A E,\|\cdot\|),(A E,||$.$) ,$ $(E A,\|\cdot\|)$ and $(E A,||$.$) are all Banach spaces, the norm inequality above and$ the Open Mapping Theorem imply that $\|$.$\| and |$.$| are equivalent on A E$ and $E A$. But $E$ is full in $\left(A,\|\cdot\|^{\prime}\right)$ by the proposition, and $\|\cdot\|^{\prime}$ is equivalent to $|$. on $A$. Therefore $E$ is full in $(A,\|\cdot\|)$.

Of course, Corollary 2.3 can be proved directly without using Proposition 2.2.

Now we give some examples.

Example 2.4. Let $A$ be a subalgebra of $\mathscr{B}(X)$ such that $\mathscr{F}(X) \subset A$. Assume that the minimal left and right ideals of $A$ are complete subspaces of $A$ (this certainly holds if $\boldsymbol{A}$ is a Banach algebra). Then it follows directly from Corollary 2.3 that every m.i. of $A$ is full in $A$. In particular, if $A$ is any algebra of operators equipped with the operator norm such that $\mathscr{F}(X) \subset A$, then $A$ has this property.

Example 2.5. Assume that $X$ is the dual space of a Banach space $Z$, and let $Y$ be the canonical image of $Z$ in $Z^{* *}$ (e.g. $X=l_{1}$ and $Y=c_{0}$ ). Let $\mathscr{F}(X, Y)$ denote the algebra of operators on $X$ generated (algebraically) by

$$
\{(f \mid x): x \in X, f \in Y\} \text {. }
$$

Assume that $A$ is any subalgebra of $\mathscr{B}(X)$, equipped with the operator norm, such that $A \cap \mathscr{F}(X)=\mathscr{F}(X, Y)$. Then by Corollary 2.3, every m.i. of $A$ is full in $A$.

Example 2.6. Let $A$ be an annihilator Banach algebra as defined by $\mathrm{F}$. F. Bonsall and A. W. Goldie in (7). If $e$ is a m.i. of $A$, then by (7, Theorem 5), 
$e$ is contained in a minimal closed ideal $M$ of $A$. Then as shown in (7, Theorem 10 ), there is an isomorphism of $M$ on to an algebra of bounded operators $B$ on a Banach space $X$ such that $\mathscr{F}(X) \subset B$. It follows, as noted in Example 2.4, that $e$ is full in $M$, and hence in $A$.

As noted in (7), the dual Banach algebras introduced by I. Kaplansky are examples of annihilator algebras. Specific examples of dual (and hence, annihilator) algebras are $H^{*}$-algebras (14, Corollary, p. 697), and $L^{p}(G)$, where $G$ is a compact topological group and $1 \leqq p<\infty$ (14, Theorem 15).

Example 2.7. Let $A$ be a $B^{*}$-algebra. By (15, Theorem 2.1), the closure of $S_{A}$ in $A$ is a dual algebra. Then Example 2.6 implies that every m.i. of $A$ is full in $A$.

Example 2.8. Assume that $e$ is a m.i. of $A$ such that $A e$ is finite dimensional. Then clearly $e$ is full in $A$. If $A$ is a $C C$ algebra as defined by I. Kaplansky in (14), then every minimal left ideal of $A$ is finite dimensional (14, Lemma 4).

Now we give an example of a primitive Banach algebra of operators with the property that no m.i. of the algebra is full in the algebra.

Example 2.9. Let $A$ be the algebra of all Fredholm integral operators on $C[0,1]$. If $T \in A$, then $T$ acts on a function $f \in C[0,1]$ according to the formula

$$
(T f)(x)=\int_{0}^{1} K(x, t) f(t) d t,
$$

where $K(x, t)$ is a continuous complex valued function on $[0,1] \times[0,1]$ Let

$$
\|T\|=\sup \{|K(x, t)|: 0 \leqq x, t \leqq 1\} .
$$

Then $A$ is a Banach algebra in the norm $\|\cdot\|$.

In what follows we use the notation

and

$$
\langle f, g\rangle=\int_{0}^{1} f(x) g(x) d x
$$

$$
\|f\|_{1}=\int_{0}^{1}|f(x)| d x .
$$

If $h, k \in C[0,1]$, let $(h \mid k)$ be the operator in $A$ defined by

Note that

$$
(h \mid k)(f)(x)=\langle h, f\rangle k(x), f \in C[0,1] \text {. }
$$

$$
\|(h \mid k)\|=\|h\|_{\infty}\|k\|_{\infty} .
$$

Now fix $h, k \in C[0,1]$ and assume that $\langle h, k\rangle=1$. Then $E=(h \mid k)$ is a m.i. of $A$. If $K(x, t)$ is continuous on $[0,1] \times[0,1]$, and $S$ is the Fredholm integral operator determined by $K(x, t)$, then let

$$
\left(S^{\prime} f\right)(x)=\int_{0}^{1} K(t, x) f(t) d t, f \in C[0,1] .
$$


If $S, T \in A$, we have

and

$$
E S=\left(S^{\prime} h \mid k\right), T E=(h \mid T k),
$$

$$
\begin{aligned}
(T E, E S) E & =E S T E=\left(S^{\prime} h \mid k\right)(h \mid T k) \\
& =\left\langle T k, S^{\prime} h\right\rangle E .
\end{aligned}
$$

Then $\|E S\|=\left\|S^{\prime} h\right\|_{\infty}\|k\|_{\infty}$, so that

$$
\begin{aligned}
|(T E, E S)| & =\left|\left\langle T k, S^{\prime} h\right\rangle\right| \leqq\left\|S^{\prime} h\right\|_{\infty}\|T k\|_{1} \\
& \leqq\|E S\|\|T k\|_{1}\|k\|_{\infty}^{-1} .
\end{aligned}
$$

If $E$ were full in $A$, there would exist $J>0$ such that

$$
\|T E\| \leqq J \sup \{|(T E, E S)|:\|E S\|=1\}
$$

for all $T \in A$. Then there would exist $M>0$ such that

$$
\|T k\|_{\infty}\|h\|_{\infty} \leqq M\|T k\|_{1}
$$

for all $T \in A$. But since $\{T k: T \in A\}=C[0,1]$, this is a contradiction. Therefore $E$ is not full in $A$.

We close this section with several remarks concerning the case when $A$ is a primitive algebra.

Remark 2.10. Let $A$ be a primitive algebra with the property that every minimal left ideal and every minimal right ideal of $A$ are complete subspaces of $A$. Let $e$ and $f$ be m.i.'s of $A$ and assume that $e$ is full in $A$. We show that $f$ must also be full in $A$. Suppose $f A e=\{0\}$. Then $(A f)(A e)=\{0\}$ and $\{0\}$ is a primitive ideal by hypothesis. Therefore by (18, Theorem (2.2.9) (iv)), either $A f=\{0\}$ or $A e=\{0\}$, a contradiction. Thus we can choose $u \in A$ such that $f u e \neq 0$. The map $b f \rightarrow b f u e$ is a continuous isomorphism of $A f$ onto $A e$. By the Open Mapping Theorem there exists $K>0$ such that $K\|b f u e\| \geqq\|b f\|$ for all $b \in A$. Similarly, there exists $v \in A$ such that $f a \rightarrow e v f a$ is a bicontinuous isomorphism of $f A$ onto $e A$, and there exists $J>0$ such that $J\|e v f a\| \geqq\|f a\|$ for all $a \in A$. Also, there exists $M>0$ such that for all $b \in A$,

$$
\|b e\| \leqq M \sup \{|(b e, e a)|:\|e a\| \leqq 1\} .
$$

Then for all $b \in A$,

Note that

$$
\begin{aligned}
\|b f\| & \leqq K \| \text { bfue } \| \\
& =K M \sup \{\mid(b f u e, \text { evfa }) \mid: \| \text { evfa } \| \leqq 1\} \\
& \leqq K M J \sup \{\mid b f u e, \text { evfa }) \mid:\|f a\| \leqq 1\} .
\end{aligned}
$$

$$
\begin{aligned}
|(b f u e, e v f a)|\|e\| & =\|e v(f a b f) u e\| \\
& \leqq \| \text { evfue } \||(b f, f a)| .
\end{aligned}
$$


Thus for all $b \in A$,

$$
\|b f\| \leqq Q \sup \{|(b f, f a)|:\|f a\| \leqq 1\} \text {, for some } Q>0 .
$$

Remark 2.11. Let $A$ be primitive. Let $e$ be a m.i. of $A$. Define an algebra norm $\|\cdot\|^{\prime}$ on $A$ by

$$
\|a\|^{\prime}=\sup \{\|e b a\|:\|e b\| \leqq 1\} .
$$

Note that $\|a\|^{\prime} \leqq\|a\|, a \in A$. Then

$$
\begin{aligned}
\|a e\|^{\prime} & =\sup \{\|e b a e\|:\|e b\| \leqq 1\} \\
& =\|e\| \sup \{|(a e, e b)|:\|e b\| \leqq 1\} \\
& \leqq\|e\| \sup \left\{|(a e, e b)|:\|e b\|^{\prime} \leqq 1\right\} .
\end{aligned}
$$

Therefore $e$ is full in $\left(A,\|\cdot\|^{\prime}\right)$.

\section{Representations of algebras with minimal left ideals}

Throughout this section $\pi$ is a continuous representation of $A$ into $\mathscr{B}(X)$. We use the notation

$$
Z_{\pi}=\left\{x \in X: \pi\left(S_{A}\right) x=\{0\}\right\} .
$$

If $Y$ is a $\pi$-invariant subspace of $X$, we denote the restriction of $\pi$ to $Y$ by $\pi \mid Y$ (i.e. $\pi \mid Y$ is the representation of $A$ on $Y$ defined by $(\pi \mid Y)(a) y=\pi(a) y$ for $a \in A, y \in Y$ ).

In this section we prove our main results concerning representations of algebras with minimal left ideals. We begin with two lemmas.

Lemma 3.1. Let e be a m.i. of $A$. Fix $x_{1} \in \mathscr{R}(\pi(e)), x_{1} \neq 0$, and set

Then

$$
X_{1}=\operatorname{cl}\left(\pi(A) x_{1}\right) \text {. }
$$

(1) if $X_{1} \cap Z_{\pi}=\{0\}$, then $\pi(A)$ acts irreducibly on $X_{1}$, and

(2) if the minimal left ideal Ae is a complete subspace of $A$, and if e is full in $A$, then $X_{1}$ is bicontinuously isomorphic to Ae, $\pi(A)$ acts strictly irreducibly on $X_{1}$, and $\pi \mid X_{1}$ is equivalent to the left regular representation of $A$ on Ae.

Proof. First we verify that $\pi(A)$ acts strictly irreducibly on $\pi(A) x_{1}$. Assume $y_{1}, y_{2} \in \pi(A) x_{1}$ and $y_{1} \neq 0$. Then there exist $a_{k} \in A$ such that $y_{k}=\pi\left(a_{k}\right) x_{1}$, $k=1,2$. Since $a_{1} e \neq 0, A a_{1} e=A e$. Therefore there exists $b \in A$ such that $b a_{1} e=a_{2} e$. Then $\pi(b) y_{1}=\pi(b) \pi\left(a_{1}\right) x_{1}=\pi\left(a_{2}\right) x_{1}=y_{2}$.

Assume that $W$ is a closed $\pi$-invariant subspace of $X$ such that $W \subset X_{1}$. Then since $\pi(A)$ acts strictly irreducibly on $\pi(A) x_{1}$, either $W \cap\left(\pi(A) x_{1}\right)=\{0\}$ or $\pi(A) x_{1} \subset W$. In the latter case, $W=X_{1}$. Assume $W \cap\left(\pi(A) x_{1}\right)=\{0\}$. Denote by $\left\langle x_{1}\right\rangle$ the span of $x_{1}$ in $X$. Then for all $a \in A, \pi(e a) X_{1} \subset\left\langle x_{1}\right\rangle$. 
Therefore $\pi(A e A) W \subset W \cap\left(\pi(A) x_{1}\right)=\{0\}$. If $f$ is any m.i. of $A$, then either $f \in A e A$ or $f A e=\{0\}$. Thus $\pi(f) W=\{0\}$ in either case. Therefore

$$
\pi\left(S_{A}\right) W=\{0\},
$$

so that $W \subset Z_{\pi}$. Then assuming that $X_{1} \cap Z_{\pi}=\{0\}$, we have $W=\{0\}$. This proves (1).

Now assume that $A e$ is complete and $e$ is full in $A$. Consider the continuous map of $A e$ onto $\pi(A) x_{1}$ defined by $a e \rightarrow \pi(a e) x_{1}=\pi(a) x_{1}$. Since $e$ is full in $A$, there exists $J>0$ such that for any element $a e \in A e$, there exists $e b \in e A$ such that $\|e b\| \leqq J$ and $e b a e=\|a e\| e$. If $a e \in A e$ and $e b$ is chosen as above, then

$$
\begin{aligned}
\|a e\|\left\|x_{1}\right\| & =\left\|\pi(e b a e) x_{1}\right\| \\
& \leqq\|\pi\|\|e b\|\left\|\pi(a) x_{1}\right\| \\
& \leqq(\|\pi\| J)\left\|\pi(a) x_{1}\right\| .
\end{aligned}
$$

This proves that the map $a e \rightarrow \pi(a) x_{1}$ is a bicontinuous isomorphism of $A e$ on to $\pi(A) x_{1}$. Since $A e$ is complete, $X_{1}=\operatorname{cl}\left(\pi(A) x_{1}\right)=\pi(A) x_{1}$. Denote the map $a e \rightarrow \pi(a) x_{1}$ by $V$. If $b \in A$, let $\gamma(b)$ be the operator defined on $A e$ by left multiplication by $b$. Then $b \rightarrow \gamma(b)$ is the left regular representation of $A$ on $A e$. We have

$$
\begin{aligned}
\left(V^{-1} \pi(b) V\right) a e & =V^{-1} \pi(b) \pi(a) x_{1} \\
& =V^{-1} \pi(b a) x_{1} \\
& =b a e \\
& =(\gamma(b)) a e .
\end{aligned}
$$

Therefore the restriction of $\pi$ to $X_{1}$ is equivalent to the left regular representation of $A$ on $A e$. This proves (2).

Lemma 3.2. Let $e$ be a full m.i. of $A$. Fix $x_{1} \in \mathscr{R}(\pi(e)), x_{1} \neq 0$, and let $X_{1}=\operatorname{cl}\left(\pi(A) x_{1}\right)$.

Then there exists a closed $\pi$-invariant subspace $X_{2}$ such that $X_{1} \cap X_{2}=\{0\}$, $X_{1}+X_{2}$ is closed in $X$, and $\pi\left(S_{A}\right) X \subset X_{1}+X_{2}$.

Proof. Choose $M$ a closed complement of the span of $x_{1}$ in $\mathscr{R}(\pi(e))$. Let

$$
X_{2}=\operatorname{cl}(\pi(A) M+\pi(P) X)
$$

where $P=\{a \in A: e A a=\{0\}\}$. Suppose that there exists $\left\{a_{n}\right\} \subset A$ and $\left\{w_{n}\right\} \subset X_{2}$ such that $\left\|\pi\left(a_{n}\right) x_{1}\right\|=1, n \geqq 1$, and

$$
\left\|\pi\left(a_{n}\right) x_{1}+w_{n}\right\|=\varepsilon_{n} \rightarrow 0 .
$$

There exists $K>0$ such that

$$
K\left\|a_{n} e\right\| \geqq\left\|\pi\left(a_{n}\right) x_{1}\right\|=1, \quad n \geqq 1 .
$$


Also, since $e$ is full in $A$, there exists $J>0$ and $\left\{b_{n}\right\} \subset A$ such that $\left\|e b_{n}\right\| \leqq J$ and $\left(a_{n} e, e b_{n}\right) e=e b_{n} a_{n} e=\left\|a_{n} e\right\| \mathrm{e}, n \geqq 1$. Therefore

$$
\begin{aligned}
& \left\|x_{1}+\right\| a_{n} e\left\|^{-1} \pi\left(e b_{n}\right) w_{n}\right\| \\
& \quad=\left\|\left(\left\|a_{n} e\right\|^{-1} \pi\left(e b_{n} a_{n} e\right) x_{1}\right)+\left(\left\|a_{n} e\right\|^{-1} \pi\left(e b_{n}\right) w_{n}\right)\right\| \\
& \quad \leqq(K J\|\pi\|) \varepsilon_{n} \rightarrow 0 .
\end{aligned}
$$

Note that for any $b \in A, \pi(e b) X_{2} \subset M$. Thus

$$
u_{n}=\left\|a_{n} e\right\|^{-1} \pi\left(e b_{n}\right) w_{n} \in M
$$

for $n \geqq 1$, and $\left\|x_{1}+u_{n}\right\| \rightarrow 0$. This contradicts the choice of $M$. It follows that there exists $\varepsilon>0$ such that $\left\|y_{1}+y_{2}\right\| \geqq \varepsilon$ whenever $y_{1} \in X_{1},\left\|y_{1}\right\|=1, y_{2} \in X_{2}$. Also, both $X_{1}$ and $X_{2}$ are closed in $X$ by definition. These facts imply by a standard argument that $X_{1}+X_{2}$ is closed in $X$ and that $X_{1} \cap X_{2}=\{0\}$.

Now assume that $a, b \in A$ and $x \in X$. Then $\pi(e) \pi(a) x=\lambda x_{1}+x_{2}$ for some scalar $\lambda$ and some vector $x_{2} \in M$. Then $\pi(b e a) x=\lambda \pi(b) x_{1}+\pi(b e) x_{2} \in X_{1}+X_{2}$. Therefore $\pi(A e A) X \subset X_{1}+X_{2}$. Also, from the definition of $X_{2}$ we have $\pi(P) X \subset X_{2}$. If $f$ is a m.i. of $A$ and $f \notin P$, then $A e A f \neq\{0\}$. Then

$$
A f=(A e A) f \subset A e A \text {. }
$$

Thus either $f \in P$ or $f \in A e A$. It follows that $S_{A} \subset A e A+P$. These arguments prove that $\pi\left(S_{A}\right) X \subset X_{1}+X_{2}$.

Let $\ell_{\pi}$ be the collection of all closed $\pi$-invariant subspaces of $X$ that are not included in $Z_{\pi}$. The set $\ell_{\pi}$ is partially ordered by inclusion.

Now we prove the main result of this section.

Theorem 3.3. Let $A$ be a semisimple normed algebra with the property that every m.i. of $A$ is full in $A$. Let $\pi: A \rightarrow \mathscr{B}(X)$ be a continuous representation of $A$ such that $\operatorname{cl}\left(\pi\left(S_{A}\right) X\right)=X$. Then:

(1) If $Y$ is a closed $\pi$-invariant subspace of $X$ and $Y \cap Z_{\pi}=\{0\}$, then there exists a minimal subspace $X_{1} \in \ell_{\pi}$ such that $X_{1} \subset Y$. When the minimal left ideals of $A$ are complete subspaces of $A$, the same conclusion holds whenever $Y \in \ell_{\pi}$.

(2) If the minimal left ideals of $A$ are complete subspaces of $A$, and if $X_{1}$ is a minimal subspace in $\ell_{\pi}$, then $X_{1}$ is bicontinuously isomorphic to some minimal left ideal Ae of $A$, and $\pi \mid X_{1}$ is equivalent to the left regular representation of $A$ on Ae.

(3) If the minimal left ideals of $A$ are complete, then $X$ is the topological sum of the collection of subspaces in $\ell_{\pi}$ which are minimal in $\ell_{\pi}$.

(4) Every minimal subspace in $\ell_{\pi}$ has a closed $\pi$-invariant complement in $X$.

Proof. Assume that $Y$ is a closed $\pi$-invariant subspace of $X$ such that $Y \not \subset Z_{\pi}$. Then there exist $x \in Y$ and a m.i. $e$ of $A$ such that $\pi(e) x \neq 0$. Let $x_{1}=\pi(e) x \in Y$. Then $\pi(A) x_{1} \subset Y$, and setting $X_{1}=\operatorname{cl}\left(\pi(A) x_{1}\right)$, (1) follows directly from Lemma 3.1.

Assume now that the hypothesis in (2) is satisfied and that $X_{1}$ is a minimal subspace in $\ell_{\pi}$. By the previous argument and Lemma 3.1, $X_{1}=\pi(A e) x_{1}$ for 
some m.i. $e$ of $A$ and some $x_{1} \in X_{1}$. Then (2) follows from Lemma 3.1.

If every minimal left ideal of $A$ is complete, then by Lemma 3.1 every nonzero subspace of $X$ of the form $\pi(A e) x$ where $e$ is a m.i. of $A, x \in X$, is a closed minimal $\pi$-invariant subspace of $X$. If $y \in \pi\left(S_{A}\right) X$, then

$$
y=\pi\left(a_{1} e_{1}\right) x_{1}+\ldots+\pi\left(a_{n} e_{n}\right) x_{n}
$$

where for each $k, 1 \leqq k \leqq n, e_{k}$ is a m.i. of $A, a_{k} \in A$, and $x_{k} \in X$. Thus $y \in \pi\left(A e_{1}\right) x_{1}+\ldots+\pi\left(A e_{n}\right) x_{n}$. Therefore $\pi\left(S_{A}\right) X$ is included in the sum of the collection of subspaces in $\ell_{\pi}$ which are minimal in $\ell_{\pi}$. This proves (3).

(4) is a direct consequence of Lemma 3.2.

If $e$ is a m.i. of $A$ and $e$ is not full in $A$, then the conclusions of Theorem 3.3 need not hold. We illustrate this with two examples.

Example 3.4. Let $A$ be a normed algebra, and assume that $e$ is a m.i. of $A$ such that $e$ is not full in $A$. Also, assume $A e$ is a complete subspace of $A$. Norm $A e$ with the norm

$$
\|a e\|_{1}=\sup \{|(a e, e b)|:\|e b\| \leqq 1\} .
$$

Since $e$ is not full in $A$, then the two norms $\|\cdot\|$ and $\|\cdot\|_{1}$ are not equivalent on $A e$. Let $X_{1}$ be the completion of $\left(A e,\|\cdot\|_{1}\right)$. Let $\gamma$ denote the left regular representation of $A$ on $A$ e, i.e.

$$
\gamma(a)(b e)=a b e, b e \in A e .
$$

Then if $a \in A$, be $\in A e$, we have

$$
\begin{aligned}
&\|\gamma(a) b e\|_{1}=\sup \{|(a b e, e c)|:\|e c\| \leqq 1\} \\
&=\|e\|^{-1} \sup \left\{\left(\frac{\|e c a b e\|}{\|e c a\|}\right)\left(\frac{\|e c a\|}{\|e c\|}\right): e c a \neq 0\right\} \\
& \leqq\|a\| \sup \{|(b e, e c a)|:\|e c a\| \leqq 1\} \\
&=\|a\|\|b e\|_{1} .
\end{aligned}
$$

Thus $a \rightarrow \gamma(a)$ extends to a continuous representation of $A$ into $\mathscr{B}\left(X_{1}\right)$. This extended representation is not equivalent to $\gamma$, in contrast to the conclusion of Theorem 3.3(2). (Note: $\gamma$ is strictly irreducible on $A e$, while the extension of $\gamma$ is not strictly irreducible on $X_{1}$ ).

Example 3.5. Let $X$ be a Banach space and assume that $X_{1}$ and $X_{2}$ are closed subspaces of $X$ with the properties

(i) $X_{1} \cap X_{2}=\{0\}$,

(ii) $X_{1}+X_{2}$ is not closed in $X$, and

(iii) $X_{1}+X_{2}$ is dense in $X$.

If $X$ is a separable Hilbert space, it is shown in (11, pp. 28-29) that there exist subspaces $X_{1}$ and $X_{2}$ with these properties. Let

and let

$$
\mathscr{F}_{1}=\left\{(f \mid x): x \in X_{1}, f\left(X_{2}\right)=\{0\}\right\},
$$

$$
\mathscr{F}_{2}=\left\{(f \mid x): x \in X_{2}, f\left(X_{1}\right)=\{0\}\right\} \text {. }
$$


Note that the subspaces $X_{1}$ and $X_{2}$ are invariant for both $\mathscr{F}_{1}$ and $\mathscr{F}_{2}$. Let $A$ be the closed subalgebra of $\mathscr{B}(X)$ generated by $\mathscr{F}_{1}$ and $\mathscr{F}_{2}$. Then $X_{1}$ and $X_{2}$ are invariant for $A$.

If $x, y \in X_{1}$ and $x \neq 0$, there exists $f \in X^{*}$ such that $f\left(X_{2}\right)=\{0\}$ and $f(x)=1$. Then $(f \mid x) \in \mathscr{F}_{1}$ and $(f \mid y)(x)=y$. Thus $\mathscr{F}_{1}$ acts strictly irreducibly on $X_{1}$, and similarly, $\mathscr{F}_{2}$ acts strictly irreducibly on $X_{2}$. Let

$$
P_{k}=\left\{T \in A: T\left(X_{k}\right)=0\right\}, k=1,2 .
$$

Then $P_{k}$ is primitive for $k=1,2$, and by (iii), $P_{1} \cap P_{2}=\{0\}$. Thus $A$ is semisimple.

Now we verify that $X_{1}$ has no closed $A$-invariant complement in $X$. For suppose $X=X_{1} \oplus Y$ where $Y$ is a closed $A$-invariant subspace of $X$. If $x_{2} \in X_{2}$, then $x_{2}=x_{1}+y$ for some $x_{1} \in X_{1}, y \in Y$. Choose $T \in \mathscr{F}_{1}$ such that $T x_{1}=x_{1}$ and $T x_{2}=0$. Then $0=x_{1}+T y$, so that $x_{1}=T y=0$. Therefore $x_{2}=y$. This proves that $X_{2} \subset Y$. Now there exists $K>0$ such that

$$
K\|x+y\| \geqq\|x\|
$$

whenever $x \in X, y \in Y$. But then this same inequality holds whenever $x \in X_{1}$, $y \in X_{2}$. This contradicts (ii). Now let $E=(f \mid x) \in \mathscr{F}_{1}$ where $f(x)=1$. Then $E$ is a minimal idempotent of $A$ and the map $T E \rightarrow T X$ maps $A E$ on to $X_{1}$. However, $X_{1}$ has no closed $A$-invariant complement.

\section{The structure of representations}

Let $\pi: A \rightarrow \mathscr{B}(X)$ be a continuous representation of $A$. Let $e$ be a m.i. of $A$, and let $I$ be the minimal closed ideal $\mathrm{cl}(A e A)$. We denote by $\pi_{I}$ the restriction of $\pi$ to $X_{1}=\operatorname{cl}(\pi(I) X)$. In the first part of this section we describe the representations $\pi_{I}$. Later in the section we show how in some cases $\pi$ can be written as a sum of the representations $\left\{\pi_{I}: I\right.$ a minimal closed ideal of $\left.A\right\}$.

Before describing the general form of $\pi_{l}$, we consider the case where $\pi(e)$ has finite dimensional range. In this case, assuming that $e$ is full in $A$, the structure of $\pi_{I}$ is especially simple. The key result is the following proposition. This proposition is essentially contained in P. Chernoff's paper (8) in the case where $A$ is an algebra of operators on a Banach space $Y$ such that $\mathscr{F}(Y) \subset A$.

Proposition 4.1. Assume that $e$ is a m.i. of $A$ which is full in $A$, that Ae is complete, and that $\mathscr{R}(\pi(e))$ is finite dimensional. Let $\left\{x_{1}, \ldots, x_{n}\right\}$ be a basis for $\mathscr{R}(\pi(e))$.

Then $\pi(A e A) X$ is a closed subspace of $X$ and is the finite direct sum of the closed subspaces $\pi(A) x_{k}, 1 \leqq k \leqq n$.

Proof. Assume that $\pi\left(b_{1}\right) x_{1}+\ldots+\pi\left(b_{n}\right) x_{n}=0$, and suppose that

$$
\pi\left(b_{j}\right) x_{j} \neq 0
$$

for some $j$. We may assume $j=1$. Choose $a \in A$ such that $e a b_{1} e=e$. Then

so that

$$
\pi\left(e a b_{1} e\right) x_{1}+\pi\left(e a b_{2} e\right) x_{2}+\ldots+\pi\left(e a b_{n} e\right) x_{n}=0 \text {, }
$$

$$
x_{1}+\lambda_{2} x_{2}+\ldots+\lambda_{n} x_{n}=0
$$


for some scalars $\lambda_{k}$. This is a contradiction. Thus the subspaces $\pi(A) x_{k}$ are algebraically independent.

Next we prove that $\pi(A e A) X=\pi(A) x_{1}+\ldots+\pi(A) x_{n}$. For if $a, b \in A$ and $x \in X$, then $\pi(e b) x=\lambda_{1} x_{1}+\ldots+\lambda_{n} x_{n}$ for some scalars $\lambda_{k}$. Then

$$
\pi(a e b) x \in \pi(A) x_{1}+\ldots+\pi(A) x_{n}
$$

which proves the assertion.

By Lemma 3.1 each of the subspaces $\pi(A) x_{k}$ is closed. Let

$$
W=\operatorname{cl}\left(\pi(A) x_{1}+\ldots+\pi(A) x_{n}\right) .
$$

By Lemma 3.2 and its proof there exist continuous projections $P_{k}$ on the Banach space $W$ such that $P_{k}$ maps $W$ on to $\pi(A) x_{k}, l \leqq k \leqq n$, and $P_{k}\left(\pi(A) x_{j}\right)=\{0\}$ if $k \neq j$. Fix $w \in W$ and choose $\left\{w_{m}\right\}$ in the subspace $\pi(A) x_{1}+\ldots+\pi(A) x_{n}$ such that $w_{m} \rightarrow w$. Then for each $m, w_{m}=P_{1}\left(w_{m}\right)+\ldots+P_{n}\left(w_{m}\right)$, so that letting $m \rightarrow \infty$, we have $w=P_{1}(w)+\ldots+P_{n}(w)$. This proves that

$$
W=\pi(A) x_{1}+\ldots+\pi(A) x_{n}=\pi(A e A) X .
$$

Let $e$ be a m.i. of $A$, and let $A e^{(n)}$ be the linear space of $n$-tuples

$$
\left\{\left(a_{1} e, \ldots, a_{n} e\right): a_{k} \in A\right\}
$$

(algebraic operations are performed coordinatewise) and with norm

$$
\left\|\left(a_{1} e, \ldots, a_{n} e\right)\right\|=\left\|a_{1} e\right\|+\ldots+\left\|a_{n} e\right\| .
$$

Let $\gamma^{(n)}$ be the representation of $A$ defined on $A e^{(n)}$ by

$$
\gamma^{(n)}(a)\left(a_{1} e, \ldots, a_{n} e\right)=\left(a a_{1} e, \ldots, a a_{n} e\right) .
$$

Corollary 4.2. Assume the hypotheses of Proposition 4.1 hold. Let I be the minimal closed ideal $\mathrm{cl}(A e A)$. Then $\pi_{I}$ is equivalent to $\gamma^{(n)}$ on $A e^{(n)}$.

Proof. Let $\left\{x_{1}, \ldots, x_{n}\right\}$ be a basis for $\mathscr{R}(\pi(e))$. Then by Proposition 4.1,

Define

$$
\operatorname{cl}(\pi(I) X)=\pi(A e A) X=\pi(A) x_{1} \oplus \ldots \oplus \pi(A) x_{n} .
$$

$$
V\left(a_{1} e, \ldots, a_{n} e\right)=\pi\left(a_{1}\right) x_{1}+\ldots+\pi\left(a_{n}\right) x_{n} .
$$

An elementary computation verifies that $\pi_{I}=V \gamma^{(n)} V^{-1}$.

Corollary 4.3. Let $A$ be an algebra such that every minimal left ideal of $A$ is complete and every m.i. of $A$ is full in $A$. Let $\pi$ be a continuous representation of $A$ on $X$ such that $\pi\left(S_{A}\right) \subset \mathscr{K}(X)$. If I is a minimal closed ideal of $A$ that contains a m.i. e such that $\pi(e) \neq 0$, then $\pi_{I}$ is equivalent to $\gamma^{(n)}$ on $A e^{(n)}$ for some integer $n$.

Proof. The projection $\pi(e)$ is a compact operator and therefore must have finite dimensional range. Thus the result follows from the previous corollary.

Now we consider the representations $\pi_{I}$ in the general case. Our treatment essentially follows Chernoff's description of the representations of $\mathscr{F}(Y)$ (8, Section 2). 
Let $e$ be a m.i. of $A$, and let $\pi$ be a continuous representation of $A$ into $\mathscr{B}(X)$ with $\pi(e) \neq 0$. Set $M=\mathscr{R}(\pi(e))$. Define $W_{0}: A e \times M \rightarrow X$ by

$$
W_{0}(a e, m)=\pi(a) m, a \in A, m \in M \text {. }
$$

$W_{0}$ is a bilinear map of $A e \times M$ into $X$. Then $W_{0}$ extends to a linear map $W: A e \otimes M \rightarrow X$ such that $W(a e \otimes m)=W_{0}(a e, m)=\pi(a) m$.

Let $\gamma$ denote the left regular representation of $A$ on $A e$.

(1) $W(\gamma(b) \otimes 1)=\pi(b) W$ on $A e \otimes M$ for all $b \in A$.

To prove (1), let $a e \otimes m$ be an elementary tensor in $A e \otimes M$. Then

(2) $W$ is one-to-one on $A e \otimes M$.

$$
\begin{aligned}
W(\gamma(b) \otimes 1)(a e \otimes m) & =W(b a e \otimes m) \\
& =\pi(b) \pi(a e) m \\
& =\pi(b) W(a e \otimes m) .
\end{aligned}
$$

For suppose that $t=a_{1} e \otimes m_{1}+\ldots+a_{n} e \otimes m_{n}$ where $\left\{a_{1} e, \ldots, a_{n} e\right\}$ is linearly independent in $A e$ and $W(t)=0$. By the Density Theorem for Irreducible Modules (13, p. 28), for each $j, 1 \leqq j \leqq n$, there exists $b_{j} \in A$ such that $b_{j} a_{j} e=e$ and $b_{j} a_{k} e=0$, if $k \neq j$. (Note: in applying the Density Theorem, $A e$ is a strictly irreducible left $A$-module and the centraliser $\Gamma$ of $A e$ is the collection of linear operators that act on $A e$ by right multiplication by elements of the form ebe, $b \in A$. Thus in our case, $\Gamma$ is isomorphic to the complex numbers.) Then

Therefore $t=0$.

$$
\begin{aligned}
0 & =\pi\left(b_{j}\right) W(t)=W\left(\gamma\left(b_{j}\right) \otimes 1\right)(t) \\
& =W\left(e \otimes m_{j}\right)=m_{j}, \quad 1 \leqq j \leqq n .
\end{aligned}
$$

Define a function $\|\cdot\|_{\pi}$ on $A e \otimes M$ by $\|t\|_{\pi}=\|W(t)\|$. By (2), $\|\cdot\|_{\pi}$ is a norm on $A e \otimes M$. Let $A e \otimes_{\pi} M$ be the completion of $A e \otimes M$ with respect to the norm $\|\cdot\|_{\pi}$.

(3) $W$ extends to an isometry $V$ of $A e \otimes_{\pi} M$ onto $X_{1}=\operatorname{cl}(\pi(A e A) X)$.

To prove (3), first note that by definition $\|W(t)\|=\|t\|_{\pi}$ for all $t \in A e \otimes M$. Thus $W$ extends uniquely to an isometry $V: A e \otimes_{\pi} M \rightarrow X$. Also,

$$
\mathscr{R}(V)=\operatorname{cl}(\mathscr{R}(W)) \text {, }
$$

and $\mathscr{R}(W)=\pi(A) M$. Therefore

$$
\pi(A e A) X=\pi(A) \pi(e A) X=\pi(A) M=\mathscr{R}(W) .
$$

Thus $\mathscr{R}(V)=X_{1}$.

Consider the representation $\gamma \otimes 1$ acting on $A e \otimes M$. If $a \in A$ and $t \in A e \otimes M$, we have

$$
\begin{aligned}
\|(\gamma(a) \otimes 1) t\|_{\pi} & =\|W(\gamma(a) \otimes 1) t\| \\
& =\|\pi(a) W(t)\| \text { by }(1) \\
& \leqq\|\pi\|\|a\|\|W(t)\| \\
& =\|\pi\|\|a\|\|t\|_{\pi} .
\end{aligned}
$$

E.M.S. $-19 / 2-N$ 
Thus $\gamma \otimes 1$ is a continuous representation of $A$ on $\left(A e \otimes M,\|\cdot\|_{\pi}\right)$. Let $\rho$ be the unique extension of $\gamma \otimes 1$ to a continuous representation of $A$ on $A e \otimes_{\pi} M$.

Now we have all the ingredients for a characterisation (up to equivalence) of the representation $\pi_{I}$ where $I$ is the minimal closed ideal $\mathrm{cl}(A e A)$.

Theorem 4.4. The representation $\pi_{I}$ on $X_{1}=\operatorname{cl}(\pi(I) X)$ is equivalent to the representation $\rho$ on $\mathrm{Ae} \otimes_{\pi} M$.

Proof. By (3), $W$ extends to an isometry $V$ of $A e \otimes_{\pi} M$ onto $X_{1}$. By (1), $W(\gamma(b) \otimes 1)=\pi(b) W$ on $A e \otimes M$ for all $b \in A$. It follows that $V \rho(b)=\pi(b) V$ on $A e \otimes_{\pi} M$ for all $b \in A$. This proves the result.

If $e$ is a m.i. of $A$ and $M$ is a normed linear space, it is not difficult to construct a representation of $A$ having essentially the same form as the representation $\rho$ defined above. Let $\gamma$ be the left regular representation of $A$ on $A e$. Let $\|\cdot\|_{1}$ be any norm on $A e \otimes M$ such that, for some $K>0$,

$$
\|(\gamma(b) \otimes 1) t\|_{1} \leqq K\|b\|\|t\|_{1}
$$

for all $b \in A$ and all $t \in A e \otimes M$. For example, $\|\cdot\|_{1}$ might be the greatest crossnorm on $A e \otimes M$ (see $(9$, p. 373)). Then define $\tau$ to be the unique extension of $\gamma \otimes 1$ to $X$, where $X$ is the completion of $\left(A e \otimes M,\|.\|_{1}\right)$. The representation $\tau$ has the same form as the representation $\rho$. Also, we note that if $M$ is a Banach space, and if there exist $P>0, Q>0$ such that

$$
P\|m\| \leqq\|e \otimes m\|_{1} \leqq Q\|m\|
$$

for all $m \in M$, then $\mathscr{R}(\tau(e))$ in $X$ is $\{e \otimes m: m \in M\}$ which of course is isomorphic to $M$.

Corollary 4.2 and Theorem 4.4 determine the structure of $\pi_{I}$ when $I$ is a minimal closed ideal of $A$ which contains a m.i. and $\pi$ is a continuous representation of $A$ such that $\pi(I) \neq\{0\}$. In some cases the representation $\pi$ can be written as an unconditional sum of the representations

$$
\left\{\pi_{I}: I \text { a minimal closed ideal of } A\right\} \text {. }
$$

We describe the summation process in what follows.

If $Y$ is a normed linear space, $\Lambda$ is an index set, and $\left\{y_{\lambda}: \lambda \in \Lambda\right\}$ is a collection of vectors in $Y$, we write $y=\Sigma y_{\lambda}$ if the net of finite sums

$$
\left\{S_{F}=\Sigma_{\lambda \in F} y_{\lambda}: F \subset \Lambda, F \text { finite }\right\}
$$

converges to $y$ in $Y$. Also, if $J \subset A$, let

$$
R[J]=\{a \in A: J a=\{0\}\} .
$$

Now assume that $\pi$ has the property that $\operatorname{cl}\left(\pi\left(S_{A}\right) X\right)=X$. Let $I$ be a minimal closed ideal of $A$. Let $X_{1}=\operatorname{cl}(\pi(I) X)$ and $X_{2}=\operatorname{cl}(\pi(R[I]) X)$.

Proposition 4.5. If either

(1) $A=L^{p}(G), 1 \leqq p<\infty, G$ a compact topological group, or

(2) $A$ is a dual $B^{*}$-algebra, then $X=X_{1} \oplus X_{2}$. 
Proof. First assume (1). Then $I$ is finite dimensional by (14, Lemma 4). Then by (2, Lemma 6.1), $I$ has an identity $u$. Clearly, $\pi(u) x_{1}=x_{1}$ for all $x_{1} \in X_{1}$, and $\pi(u) x_{2}=0$ for all $x_{2} \in X_{2}$. If $x_{1} \in X_{1}$ and $x_{2} \in X_{2}$, then

$$
\left\|x_{1}\right\|=\left\|\pi(u)\left(x_{1}+x_{2}\right)\right\| \leqq\|\pi(u)\|\left\|x_{1}+x_{2}\right\| .
$$

Thus $X_{1}+X_{2}$ is closed, and since $\pi\left(S_{A}\right) X \subset X_{1}+X_{2}$, we have $X=X_{1} \oplus X_{2}$.

Suppose now that $A$ is a dual $B^{*}$-algebra. If $a_{1}, \ldots, a_{n}$ are in $S_{I}$, by (3, Lemma 2.3) there exists an idempotent $e \in S_{I}$ such that $e=e^{*}$ and $e a_{k}=a_{k}$, $1 \leqq k \leqq n$. Then $\|e\|=1$. Thus if $b \in R[I]$ and $x_{1}, \ldots, x_{n}, y \in X$, then

$$
\begin{aligned}
\left\|\pi\left(a_{1}\right) x_{1}+\ldots+\pi\left(a_{n}\right) x_{n}\right\| & =\left\|\pi(e)\left[\pi\left(a_{1}\right) x_{1}+\ldots+\pi\left(a_{n}\right) x_{n}+\pi(b) y\right]\right\| \\
& \leqq\|\pi\|\left\|\left(\pi\left(a_{1}\right) x_{1}+\ldots+\pi\left(a_{n}\right) x_{n}\right)+\pi(b) y\right\| .
\end{aligned}
$$

It follows from this inequality that $X=X_{1} \oplus X_{2}$.

Assume that $A$ satisfies either (1) or (2) of Proposition 4.5 and that

$$
X=\operatorname{cl}\left(\pi\left(S_{A}\right) X\right) .
$$

Let $\left\{I_{\lambda}: \lambda \in \Lambda\right\}$ be the collection of all the minimal closed ideals of $A$. By Proposition 4.5 for each $\lambda \in \Lambda$ there exists a continuous projection $P_{\lambda}$ on $X$ with range cl $\left(\pi\left(I_{\lambda}\right) X\right)$ and null space $\mathrm{cl}\left(\pi\left(R\left[I_{\lambda}\right]\right) X\right)$. For each $x \in X$, let $x_{\lambda}=P_{\lambda}(x)$. Also, let $\pi_{\lambda}$ denote the representation $\pi \mid \mathscr{R}\left(P_{\lambda}\right)=\pi_{I}$.

Now if $A$ is either a dual $B^{*}$-algebra or $L^{2}(G), G$ a compact topological group, then for any closed ideal $J$ of $A$, we have $A=J \oplus R[J]$. In particular, for each $\lambda \in \Lambda, A=I_{\lambda} \oplus R\left[I_{\lambda}\right]$. Let $Q_{\lambda}$ be the continuous projection on $A$ with range $I_{\lambda}$ and null space $R\left[I_{\lambda}\right]$. If $a \in A$, let $a_{\lambda}=Q_{\lambda}(a)$. It follows from (1, Theorem 3.5) that $a=\Sigma a_{\lambda}$ for all $a \in A$.

We use the notation and information established above in the next two propositions.

Proposition 4.6. Assume that $A$ is a $B^{*}$-algebra. Let $\pi$ be a continuous representation of $A$ into $\mathscr{B}(X)$ such that $\mathrm{cl}\left(\pi\left(S_{A}\right) X\right)=X$. Then for all $x \in X$ and all $a \in A$,

$$
x=\Sigma x_{\lambda} \text { and } \pi(a) x=\Sigma \pi_{\lambda}(a) x_{\lambda} .
$$

Proof. Let $B=\operatorname{cl}\left(S_{A}\right)$. Then by (15, Theorem 2.1) $B$ is a dual $B^{*}$-algebra. Since $B$ has a bounded approximate identity, Cohen's Factorisation Theorem (12, Theorem 32.22) implies that

$$
\{\pi(b) y: b \in B, y \in X\}=\operatorname{cl}(\pi(B) X)=X .
$$

If $x \in X$, then $x=\pi(b) y$ for some $b \in B, y \in X$. Furthermore, $b=\Sigma b_{\lambda}$. Then $x=\Sigma \pi\left(b_{\lambda}\right) y$, and $x_{\lambda}=P_{\lambda}(x)=\pi\left(b_{\lambda}\right) y$. Therefore $x=\Sigma x_{\lambda}$, and if $a \in A$,

$$
\pi(a) x=\Sigma \pi(a) x_{\lambda}=\Sigma \pi_{\lambda}(a) x_{\lambda} .
$$

Proposition 4.7. Let $G$ be a compact topological group. Let $\pi$ be a continuous representation of $L^{1}(G)$ into $\mathscr{B}(X)$ such that $\pi$ is essential (i.e. $\left.\operatorname{cl}\left(\pi\left(L^{1}(G)\right) X\right)=X\right)$. Then for all $x \in \pi\left(L^{2}(G)\right) X$ and all $f \in L^{1}(G)$,

$$
x=\Sigma x_{\lambda} \text { and } \pi(f) x=\Sigma \pi_{\lambda}(f) x_{\lambda} .
$$


Proof. Note that $\pi$ is continuous on $L^{2}(G)$ with respect to the $L^{2}$-norm. $L^{2}(G)$ is an $H^{*}$-algebra (see $(16$, p. 100)). Assume that $x=\pi(g) y$ where $g \in L^{2}(G), y \in X$. Then $g=\Sigma g_{\lambda}$, so that $x=\Sigma \pi\left(g_{\lambda}\right) y=\Sigma x_{\lambda}$. Then

whenever $f \in L^{1}(G)$.

$$
\pi(f) x=\Sigma \pi_{\lambda}(f) x_{\lambda}
$$

\section{Some applications}

Throughout this section $\pi$ is a continuous representation of $A$ into $\mathscr{B}(X)$. In the case when $A=\mathscr{B}(Y)$ for some Banach space $Y$, we also assume that $\pi$ maps the identity in $\mathscr{B}(Y)$ on to the identity in $\mathscr{B}(X)$. The aim of this section is to apply Theorem 3.3 in the case where the representation space $X$ is certain of the classical Banach spaces.

Proposition 5.1. Assume that every minimal left ideal of $A$ is complete and that every m.i. of $A$ is full in $A$. Also, assume that $\operatorname{cl}\left(\pi\left(S_{A}\right) X\right)=X$. If $X=l_{p}$, $1 \leqq p<\infty$, or $X=c_{0}$, then any minimal left ideal of $A$ not contained in $\operatorname{ker}(\pi)$ is either finite dimensional or bicontinuously isomorphic to $X$. If $X=C(K)$, $K$ a compact Hausdorff space, then any minimal left ideal of $A$ not contained in $\operatorname{ker}(\pi)$ is either finite dimensional or non-reflexive.

Proof. Let $e$ be a m.i. if $A$ such that $\pi(e) \neq 0$. Choose $x_{1} \in X$ such that $\pi(e) x_{1}=x_{1} \neq 0$. Then by Theorem $3.3 A e$ is bicontinuously isomorphic to $\pi(A) x_{1}$ and $\pi(A) x_{1}$ is a closed complemented subspace of $X$. Therefore the first conclusion of the proposition follows from (17, Theorem 1$)$, and the second from (10, Corollaire du Proposition 5).

Corollary 5.2. Assume that $Y$ is infinite dimensional and that $A$ is a subalgebra of $\mathscr{B}(Y)$ such that $\mathscr{F}(Y) \subset A$. Assume that $\pi$ is continuous with respect to the operator norm and $\operatorname{cl}(\pi(\mathscr{F}(Y)) X)=X$. If $X=l_{p}, 1 \leqq p<\infty$, or $X=c_{0}$, then $Y$ is bicontinuously isomorphic to $X$. If $X=C(K)$, then $Y$ is nonreflexive.

Proof. Consider $A$ as normed with the operator norm. Then Proposition 5.1 applies to $A$. Since every minimal left ideal of $A$ is bicontinuously isomorphic to $Y$, the corollary follows.

In some cases the hypothesis that $X=\operatorname{cl}\left(\pi\left(S_{A}\right) X\right)$ can be replaced by special hypotheses on $A$. This is true in the next result which is a generalisation of (6, Corollary 6.15). Also, Proposition 5.4 can be applied in an obvious way to replace the hypothesis that $X=\mathrm{cl}\left(\pi\left(S_{A}\right) X\right)$ in part of Corollary 5.2. Proposition 5.4 is a slight modification of a result communicated to us by the referee.

Corollary 5.3. Assume that $A=\mathscr{B}(Y)$ where $Y$ is an infinite dimensional Banach space with a symmetric basis. Let $\pi$ be a continuous representation of $A$ into $\mathscr{B}(X)$ where $X=l_{p}, 1 \leqq p<\infty$, or $X=c_{0}$. Then $Y$ is bicontinuously isomorphic to $X$. 
Proof. It follows from (6, Corollary 6.13) that $\operatorname{cl}(\pi(\mathscr{F}(Y)) X)=X$. Then Corollary 5.2 implies the result.

Proposition 5.4. Assume that $Y$ is a Banach space with the approximation property. Let $A$ be a closed subalgebra of $\mathscr{B}(Y)$ such that $\mathscr{F}(Y) \subset A$. Let $\pi$ be a continuous representation of $A$ on a reflexive Banach space $X$. Then $Z=\operatorname{cl}(\pi(\mathscr{F}(Y)) X)$ is complemented in $X$.

Proof. Since $Y$ is bicontinuously isomorphic to a closed subspace of $X$, $Y$ is reflexive. Also, by hypothesis, $Y$ has the approximation property. It follows that there exists a bounded net $\left(F_{\alpha}\right\} \subset \mathscr{F}(Y)$ which converges to the identity operator on $Y$ in the strong operator topology (see A. Grothendieck, Memoirs of the Amer. Math. Soc., Number 16, Corollaire 2, p. 181). Now for any $y \in Y, g \in Y^{*}$, we have $F_{\alpha}(g \mid y) \rightarrow(g \mid y)$ in the operator norm, so that $\pi\left(F_{\alpha}\right) \pi(g \mid y) \rightarrow \pi(g \mid y)$ in norm in $\mathscr{B}(X)$. It follows that $\pi\left(F_{\alpha}\right) \rightarrow I$ pointwise on $\pi\left(S_{A}\right) X$, and since $\left\{\pi\left(F_{\alpha}\right)\right\}$ is bounded, $\pi\left(F_{\alpha}\right) \rightarrow I$ pointwise on $Z$. By hypothesis $X$ is reflexive, and therefore closed balls in $\mathscr{B}(X)$ are compact in the weak operator topology. Thus $\left\{\pi\left(F_{\alpha}\right)\right\}$ has a cluster point $P \in \mathscr{B}(X)$. Then $P(X) \subset Z$ and $P=I$ on $Z$. Thus $P$ is a bounded projection of $X$ onto $Z$.

\section{REFERENCES}

(1) G. Bachelis, Homomorphisms of annihilator Banach algebras, Pacific $J$. Math. 25 (1968), 229-247.

(2) B. Barnes, Modular annihilator algebras, Canad. J. Math. 18 (1966), 566-578.

(3) B. Barnes, Algebras with the spectral expansion property, Illinois $J$. Math. 11 (1967), 284-290.

(4) B. Barnes, Irreducible algebras of operators which contain a minimal idempotent, Proc. Amer. Math. Soc. 30 (1971), 337-342.

(5) B. BARNes, Density theorems for algebras of operators and annihilator Banach algebras, Michigan Math. J. 19 (1972), 149-155.

(6) E. Berkson and H. Porta, Representations of $B(X), J$. Functional Analysis 3 (1969), 1-34.

(7) F. Bonsall and A. Goldie, Annihilator algebras, Proc. London Math. Soc. (3) 4 (1954), 154-167.

(8) P. Chernoff, Representations, automorphisms, and derivations of some operator algebras, J. Functional Analysis 12 (1973), 275-289.

(9) J. GIL DE LAMADRID, Topological modules: Banach algebras, tensor products, algebras of kernels, Trans. Amer. Math. Soc. 126 (1967), 361-419.

(10) A. Grothendieck, Sur les applications linéaires faiblement compactes d'espaces du type $C(K)$, Canad. J. Math. 5 (1953), 129-173.

(11) P. Halmos, Introduction to Hilbert space and the theory of spectral multiplicity (Chelsea, New York, 1951). 
(12) E. HewitT and K. Ross, Abstract harmonic analysis II (Springer-Verlag, Berlin, 1970).

(13) N. Jacobson, Structure of rings (Amer. Math. Soc. Colloq. Publ., vol. 37, Amer. Math. Soc., Providence, R.I., 1956).

(14) I. KAPLANSKY, Dual rings, Ann. of Math. (2) 49 (1948), 689-701.

(15) I. KAPLANSKY, The structure of certain operator algebras, Trans. Amer. Math. Soc. 70 (1951), 219-255.

(16) L. Looms, An introduction to abstract harmonic analysis (Van Nostrand, Princeton N.J. 1953).

(17) A. Pelczynski, Projections in certain Banach spaces, Studia Math. 19 (1960), 209-228.

(18) C. Rickart, General theory of Banach algebras (Van Nostrand, Princeton, N.J., 1960).

UNIVERSITY OF OREGON

EUGENE, OREGON 